to appear in Physica $A$

\title{
Reply to the paper
}

\section{E.G.D. Cohen, L. Rondoni: Particles, Maps and Irreversible Thermodynamics I.}

Physica A 306 (2002) 117

In [1] Cohen and Rondoni severely question the physical relevance of studies of transport by means of multibaker (MB) maps. The aim of MB studies (as well as of other work on low-dimensional chaotic models for transport) is to improve the understanding of irreversible macroscopic behavior in transport processes along one spatial dimension. These studies provide an attempt to establish a macroscopic description consistent with irreversible thermodynamics (IT) in systems with an explicit microscopic dynamics. In this class of analytically accessible model systems the properties of the microscopic and macroscopic motion are fully understood such that their mutual relation can be studied in detail. We believe that it is admissible to abandon the use of certain concepts of traditional approaches in order to highlight the general features of irreversible processes.

The fundamental interest in MB studies lies in identifying minimal assumptions on the dynamics and on the form of entropy functionals that lead to a description of the evolution of macroscopic fields according to the laws of IT - in particular, to the transport equations and to the local entropy balance. From this perspective the arguments of Cohen and Rondoni concerning the assumptions adopted in the setup of multibaker models are contradictory and part of them do not hold:

- Rondoni and Cohen [1, Sect IV] proclaim that 'it is the lack of real particles that makes local thermodynamic equilibrium (LTE) impossible'. We disagree with this statement since LTE only ascertains that the thermodynamic description of an equilibrium system applies locally in sufficiently small volumes of a non-equilibrium system. Since thermodynamics is a phenomenological theory, however, it does not involve the concept of particles. By definition of its basic objects the notion of particles can not be needed for its foundation. Hamiltonian equations of motion or kinetic theory can be used to underpin it in certain (dilute gas) limits, but IT is more general and can even describe the evolution of systems with a different underlying dynamics.

Similar to the Lorentz gas the time evolution of an ensemble of trajectories in a multibaker map can be interpreted as an assembly of non-interacting particles. By adopting a Boltzmann-Grad type limit one can then concentrate on the evolution of fields characterizing the state of the system. The characterization of these fields forms the prime objective of studies on multibaker maps. In the absence of coarse-graining the fields typically develop structures on ever refining scales as times evolves. Since their evolution is reversible and they never stop evolving, the fields are viewed as characteristics of a microscopic motion. On the other hand, the averages of the fields over MB cells of linear extension $a$ in the transport direction (called coarse-grained quantities in what follows) are thermodynamically well behaved. Appropriate averages can be interpreted as density $\varrho$, temperature $T$, and hydrodynamic velocity $v$. They are considered as local thermodynamic variables, since in a well-defined continuum limit their time evolution exactly follows the transport equations (the advection-diffusion equation, the heat conduction equation, and the Navier-Stokes equation of strongly viscous fluids) of IT. Consequently, the cell size $a$ is considered as the mesoscopic scale addressed by LTE, below which macroscopic concepts do not apply.

Information-theoretic arguments are evoked to work out the entropy balance for a coarse-grained entropy $S$ taking the form

$$
S=-k_{B} \varrho \ln \left(\varrho T^{-\gamma}\right),
$$


where $k_{B}$ is the Boltzmann constant, and $\gamma$ is a positive constant playing the role of a dimensionless specific heat. We have shown for a series of transport problems (electric [2] and heat conduction, cross effects [3], and viscous flows [ [4] that the local entropy balance of this $S$ is exactly of the form known from IT. In view of this, (1) can be interpreted as an equation of state, which holds in every cell in spite of the spatial and temporal evolution of $\varrho$ and $T$. This is in our interpretation the analog of LTE. It has nothing to do with the concept of particles and their collisions.

- We agree with Cohen and Rondoni that the expression (17) adopted for the coarse-grained entropy is not obvious, and that it is also not clear a priori how one can identify heat for a model lacking the concept of work and particles. In our early papers [2, 3] we therefore avoided the use of the term heat, and spoke mainly of entropy and entropy production. In the visco-thermal MB, on the other hand, a clear relation emerged [4. After coarse graining, the energy balance condition leads to the temperature equation

$$
\nu \varrho \partial_{x} T=\lambda \partial_{x}^{2} T+Q
$$

with

$$
Q=\nu \varrho\left(\partial_{x} v\right)^{2}
$$

where $\nu$ is the kinematic viscosity (a unique expression of the MB parameters) which coincides in the model with the heat diffusion coefficient, and $\lambda=\nu \varrho \gamma$ represents the heat conductivity. The source term $Q=\nu \varrho\left(\partial_{x} v\right)^{2}$ turns out to be the same as the contribution to the entropy production due to viscosity multiplied by the temperature. The full entropy production takes the form

$$
\sigma^{(i r r)}=\lambda\left(\frac{\partial_{x} T}{T}\right)^{2}+\frac{\nu \varrho}{T}\left(\partial_{x} v\right)^{2} .
$$

This strong analogy with IT encouraged us to call $Q$ the viscous heating term, since it indeed raises the temperature due to the local hydrodynamic momentum exchange of neighboring fluid layers, modelled by neighboring MB cells.

- Concerning the purely diffusive dynamics of the Lorentz gas or an isothermal MB, which can both be considered as particle models of transport, Rondoni and Cohen state that their entropy production only formally agrees (in an appropriate limit) with the thermodynamic expression

$$
\sigma^{(i r r)}=D \frac{(\nabla \varrho)^{2}}{\varrho}
$$

since the entropy production cannot be related to any heating process in the absence of interacting particles (in their words '... in IT the entropy production $\sigma_{s}^{I T}>0$ is due to heat, which involves randomization, ...'). We mention that IT does not predict $\sigma^{(i r r)}$ to be a source term of heat in the temperature equation. Rather [see [5] chapter XI, eq. (235)], there is no heat source at all in this equation, and local changes of the temperature are due to the Dufour effect. The assumtion of constant temperature in the diffusive multibaker only implies a vanishing Dufour coefficient. The entropy production $\sigma^{(i r r)}$ is in this case fully due to mixing entropy induced by the gradient of the macroscopic density (phase-space density in the particle picture), which is also present in microscopic models of non-interacting particles.

- The studies of MB models provide a self-consistent framework for the description of transport based on a reversible dynamics. In our eyes they clearly show that the coarse graining carried out over the underlying chaotic and mixing MB dynamics unavoidably leads to information loss, which appears to be the basic ingredient of irreversible entropy production and viscous heating. In contrast to the main assertion of [1] they therefore provide a valuable alternative approach to study the foundations of IT. 
Finally, we would like to point out that our interpretation of LTE is fully consistent with the definition given by the authors of [1], who say:

The validity of LTE can only be justified a posteriori, on the grounds of the conclusions derived from it.

We consider the recovery of the relations of IT from such a simple model as the MB map as a clear justification for considering the cell-averaged, coarse-grained fields as hallmarks of LTE, and therefore reject the criticism by Cohen and Rondoni.

[1] E. G. D. Cohen and L. Rondoni, Physica A 306, 117 (2002).

[2] J. Vollmer, T. Tél, and W. Breymann, Phys. Rev. Lett. 79(15), 2759 (1997); Phys. Rev. E 58(2), 1672 (1998); W. Breymann, T. Tél, and J. Vollmer, CHAOS 8(2), 396 (1998).

[3] L. Mátyás, T. Tél, and J. Vollmer, Phys. Rev. E 61(4), R3295 (2000), arXiv:chao-dyn/9912028; Phys. Rev. E 62(1), 349 (2000), arXiv: chao-dyn/9912034; J. Vollmer, T. Tél, and L. Mátyás, J. Stat. Phys. 101(1/2), 79 (2000), arXiv:nlin.CD/0001016.

[4] T. Tél, J. Vollmer, and L. Mátyás, Europhys. Lett. 53(4), 458 (2001), arXiv:nlin.CD/0009013; L. Mátyás, T. Tél, and J. Vollmer, Phys. Rev. E 64, 056106 (2001), arXiv:chao-dyn/0102013.

[5] S. de Groot and P. Mazur, Nonequilibrium Thermodynamics (Elsevier, Amsterdam, 1962), reprinted by Dover, New York, 1984.

László Mátyás

Max-Planck-Institute for the

Physics of Complex Systems

Nöthnitzer Str. 38

01187 Dresden

Germany
Tamás Tél

Institute for Theoretical Physics

Eötvös University

P. O. Box 32

H-1518 Budapest

Hungary
Jürgen Vollmer

Max-Planck-Institute for

Polymer Research

Ackermannweg 10

55128 Mainz

Germany 